\title{
Philonsorbonne
}

15 | 2021

Année 2020-2021

\section{Émancipation et idéologie. La phénoménologie de Ricœur à l'épreuve de la Théorie critique}

\section{Luz ASCÁRATE}

\section{(2) OpenEdition}

\section{Journals}

\section{Édition électronique}

URL : https://journals.openedition.org/philonsorbonne/1870

DOI : 10.4000/philonsorbonne. 1870

ISSN : 2270-7336

Éditeur

Publications de la Sorbonne

\section{Édition imprimée}

Date de publication : 1 janvier 2021

Pagination : 171-190

ISSN : 1255-183X

\section{Référence électronique}

Luz ASCÁRATE, «Émancipation et idéologie. La phénoménologie de Ricœur à l'épreuve de la Théorie critique », Philonsorbonne [En ligne], 15 | 2021, mis en ligne le 03 février 2021, consulté le 08 juin 2021. URL : http://journals.openedition.org/philonsorbonne/1870 ; DOI : https://doi.org/10.4000/

philonsorbonne. 1870

(c) Tous droits réservés 


\title{
Émancipation et idéologie. La phénoménologie de Ricœur à l'épreuve de la Théorie critique
}

\author{
Luz ASCÁRATE \\ ISJPS (Université Paris 1 Panthéon-Sorbonne - CNRS UMR 8103)
}

Le capitalisme tardif est une époque marquée par de multiples catastrophes : les ravages de l'exclusion, l'indifférence face à la souffrance de l'autre, les écarts économiques entre les membres des différents groupes sociaux, les conditions inhumaines de vie et de travail, le rôle dominant du spectacle et de l'informatique. Ces différentes facettes de l'objectivation, de l'instrumentalisation, de la subordination de la vie humaine aux structures statiques de la production, générant des pathologies sociales inévitables, exigent de la philosophie qu'elle adopte une orientation critique. En nous inspirant de Jean Ladrière dans "L'Abîme " ${ }^{1}$, nous pouvons identifier cette situation à une crise des fondements qui peut être comprise comme le moment historique où l'homme n'est plus considéré en tant que sujet constituant. Telle est la cause, à notre avis, de la vague antihumaniste présente dans la philosophie et les sciences humaines. À cet égard, selon Ladrière, "par une coïncidence qui tient aux conditions mêmes du développement de la problématique de la raison, trois entreprises spéculatives, très différentes cependant dans leurs inspirations respectives, ont tenté, dans les années du début de ce siècle, de mener à bien un programme de reconstruction inspiré essentiellement par l'idée de fondation: la métamathématique, l'atomisme logique, la phénoménologie $»^{2}$. Cette dernière, de manière explicite dans l'œuvre de son fondateur, cherche à faire

1. Cf. J. Ladrière, «L'Abîme », dans Savoir, faire, espérer. Les limites de la Raison, vol. 1, Bruxelles, Publications des facultés universitaires Saint-Louis, 1976.

2. Ibid., p. 171. 
face à la crise de sens de l'humanité et de la culture, y compris de la philosophie. Mais cette dernière tradition a été très critiquée par la Théorie critique, dont la première génération a dénoncé l'extrême idéalisme de la méthode phénoménologique ${ }^{3}$, qui ne nous fournirait pas la perspective nécessaire pour dégager une théorie consistante et critique du social. Face à cette apparente disjonction, la phénoménologie de l'imaginaire social de Paul Ricœur a beaucoup à dire sur la critique du social d'un point de vue constitutif. L'originalité de ce rapport est encore plus remarquable à la lumière des approches contemporaines de la question de l'émancipation. De ce fait, deux méthodes presque contraires en sont venues à se superposer à l'intérieur de la philosophie sociale ricœurienne, à savoir la critique des idéologies située dans la tradition de la Théorie critique de l'École de Francfort et la méthode phénoménologique émanant du mouvement fondé par Husserl.

En ce sens, nous montrerons dans ce qui suit que l'approche phénoménologique de Ricœur s'aligne sur la critique émancipatrice de la Théorie critique, notamment sur les développements de la dernière génération. D'un côté, Ricœur apporte une perspective du social tout à la fois constitutive, critique et centrée sur l'expérience des agents. De l'autre, ses analyses portant sur l'idéologie rendent l'entrelacement d'une perspective généalogique et d'une perspective humaniste de la critique possible. Si dans nos réflexions, nous portons surtout notre attention sur la tradition de la Théorie critique de l'École de Francfort, c'est parce que Ricœur accorde à cette tradition une place essentielle dans ses textes portant sur l'émancipation et la critique des idéologies tout en en proposant un nouveau sens.

\section{L'émancipation et la critique d'orientation constitutive}

Le concept d'émancipation occupe une place fondamentale dans le débat contemporain sur le monde social. Cependant, la tradition de la Théorie critique est la seule à avoir répondu aux demandes normatives du projet philosophique d'émancipation. En ce sens, lors des conférences données à l'Université Diego Portales de Santiago du Chili, en 2011, Luc Boltanski affirme que le programme de la sociologie critique française et celui de la Théorie critique de l'École de Francfort se sont développés en parallèle : tous deux ont en effet poursuivi un objectif d'émancipation en conjuguant marxisme et phénoménologie. Cependant, la sociologie critique française avait laissé de côté les espoirs messianiques caressés par l’École de Francfort et, contrairement à celle-ci, elle avait introduit une perspective

3. Cf. Th. Adorno, «Metakritik der Erkenntnistheorie », Gesammelte Schriften, Band. V, Francfort-sur-le-Main, Suhrkamp Verlag, 1970. 
structurale fondée sur des recherches empiriques ${ }^{4}$. La sociologie pragmatique de Boltanski vise à cet égard à renouveler d'une façon radicale la possibilité d'une sociologie critique, en cherchant à éviter de risquer d'imposer une hiérarchie verticale entre le sociologue et les individus. Plus précisément, Boltanski propose une reconstruction des critiques que les sujets sociaux peuvent émettre dans leur vie quotidienne et à partir desquelles un cadre normatif émancipateur pourrait être explicité. Le sens de l'émancipation que la sociologie pragmatique explore ne relève donc pas d'un mouvement théorique qui prolongerait le contrôle toujours plus poussé des dominés, mais de la possibilité que ceux-ci s'approprient les capacités dont ils sont privés ${ }^{5}$.

Néanmoins, la sociologie pragmatique doit résoudre au moins deux difficultés. Tout d'abord, il y a le problème du réalisme de la sociologie pragmatique, qu'il convient de résoudre en conjuguant la sociologie critique à celle pragmatique en tant que méta-critique, ainsi qu'en faisant la distinction entre monde et réalité. Ensuite, il y a le problème de l'excessive préoccupation pour la recherche empirique, qui a pour conséquence une faible justification philosophique ${ }^{6}$ des visées normatives de la critique dans la sociologie pragmatique . Face à ces deux difficultés, la phénoménologie sociale de Ricœur fournit des ressources critiques qui méritent d'être examinées avec attention et qui la rapprochent de la dernière génération de la Théorie critique.

Boltanski a lui-même reconnu l'importance de Ricœur dans la nouvelle théorie du social qu'il prend pour point de départ, et qui croise l'intention phénoménologique de revenir « aux choses elles-mêmes» à partir de la compréhension du langage propre à la philosophie analytique ${ }^{8}$. Dans la perspective ricœurienne de l'espace social, une considération originale sur l'émancipation peut précisément être identifiée, qui peut effectivement répondre aux problèmes de la critique du social.

Il ne fait aucun doute que la question de l'émancipation a constitué la tâche primordiale de l'École de Francfort depuis sa fondation. Donnons ici

4. Cf. L. Boltanski, Sociología y crítica social, Santiago de Chile, Universidad Diego Portales, 2012, p. 41. Voir aussi L. Boltanski et N. Fraser, Domination et émancipation. Pour un renouveau de la critique sociale, Lyon, Presses universitaires de Lyon, 2014.

5. L. Boltanski, De la critique. Précis de sociologie de l'émancipation, Paris, Gallimard, 2009 .

6. M. Berger, « Kritik der kritischen Kritik. Zu den philosophischen Grundlagen praxeologischer Kritik bei Pierre Bourdieu und Luc Boltanski », Allgemeine Zeitschrift für Philosophie, Vol. 39, $\mathrm{N}^{\circ} 1$ (2014).

7. T. Bogusz, «Author meets critics. Commentaire de De la critique. Précis de sociologie de l'émancipation", Centenaire du congrès national de la Deutsche Gesellschaft für Soziologie, Francfort-sur-Le-Main, 13 octobre 2010. Disponible en ligne à l'adresse suivante : https://www.academia.edu/1805345/Commentaire de Luc Boltanski De la critique . $\operatorname{Pr} \%$ C3\%A9cis dune sociologie de 1\%C3\%A9mancipation_dt. Soziologie und Sozialkritik_.

8. L. Boltanski, De la critique. Précis de sociologie de l'émancipation, op. cit. 
une précision sur cette question par rapport aux différentes générations de cette tradition. Horkheimer ${ }^{9}$, qui appartient, avec Adorno, Marcuse et Benjamin, à la génération «fondatrice » de la Théorie critique, a affirmé que celle-ci « ne vise pas seulement l'accroissement des connaissances en tant que telles, mais l'émancipation de l'homme des conditions d'esclavage $\iota^{10}$. La deuxième génération, représentée par des propositions basées sur la discussion de la proposition d'Habermas, sans pour autant laisser de côté la tâche émancipatrice, oriente la critique dans le cadre de l'action communicative. La troisième génération est représentée notamment par la pensée de Honneth. Ricœur a dialogué avec ces deux générations. Si la première s'enracinait dans la critique sociale marxiste du développement moderne de la rationalité instrumentale, à la lumière de penseurs comme Weber et Freud, la deuxième et la troisième génération continuent quant à elles à donner une place centrale à l'idée d'émancipation ${ }^{11}$, qui accompagne la préoccupation pour l'aspect normatif du monde social. Axel Honneth affirme ainsi que, "sans un concept réaliste d"intérêt émancipatoire" présumant, à des fins critiques, un noyau indestructible de disposition rationnelle chez les sujets, ce projet théorique n'aura aucun avenir $»^{2}$. Cette tâche critique comprend un aspect historique irréductible à une réflexion sur la rationalité et un regard constitutif sur l'espace social, qui oppose la Théorie critique, d'orientation normative et généalogique, aux perspectives simplement généalogiques inspirées par Foucault et à celles herméneutiques critiques inspirées par Walzer ${ }^{13}$.

L'approximation constitutive de l'espace social oppose la philosophie sociale, à laquelle appartient la Théorie critique selon Honneth, à celle politique : l'une fournit une réflexion historique et immanente des « formes de vie » sociales, l'autre donne uniquement une réflexion sur la possibilité d'une organisation démocratique qui assurerait la convivialité politique en termes de justice ${ }^{14}$. Fischbach, en suivant Honneth, insiste sur l'idée que

9. Sur le concept d'émancipation d'Horkheimer, voir notamment Katia Genel, Autorité et émancipation. Horkheimer et la Théorie critique, Paris, Payot, 2013.

10. «zielt sie nirgends bloß auf Vermehrung des Wissens als solchen ab, sondern auf die Emanzipation des Menschen aus versklavenden Verhältnissen» (M. Horkheimer, «Traditionelle und kritische Theorie (1937), Lesekreis zur Kritischen Theorie in Saarbrücken, p. 58. Disponible en ligne : http://lesekreis.blogsport.de/. Toutes les traductions ont été réalisées par nos soins.

11. A. Honneth, Ce que social veut dire, tome II : Les pathologies de la raison, trad. de l'allemand par Pierre Rusch, Paris, Gallimard, 2015.

12. A. Honneth, «Une pathologie sociale de la raison. Sur l'héritage intellectuel de la Théorie critique », La Société du mépris. Vers une nouvelle Théorie critique, trad. de l'allemand par Olivier Voirol, Pierre Rusch et Alexandre Dupeyrix, Paris, La Découverte, 2008, p. 101-130, p. 130.

13. Cf. Ibid., p. 103.

14. Cf. A. Honneth, Das Andere der Gerechtigkeit: Aufsätze zur praktischen Philosophie, Francfort-sur-le-Main, Suhrkamp Verlag, 2000. 
la philosophie sociale est le nom actuel de la pensée en quête d'émancipation. Elle réalise une description critique des dimensions pathologiques des sociétés modernes comme l'aliénation, l'idéologie, la lutte des classes, le fétichisme, le nihilisme, le désenchantement du monde, la perte de sens, la réification et l'existence inauthentique, entre autres ${ }^{15}$.

La quatrième génération de la Théorie critique se situe du côté de la philosophie sociale, qui rapproche le projet d'émancipation d'une perspective constitutive du social. Tout l'intérêt de la perspective phénoménologique de Ricœur réside précisément, dans ses apports à cette génération. L'avènement de cette dernière est marqué par des propositions formulées au début du $\mathrm{XXI}^{\mathrm{e}}$ siècle qui tentent d'établir des liens fructueux avec d'autres traditions de pensée (par exemple, avec les traditions analytique, pragmatiste, généalogiste, phénoménologique et postcoloniale, entre autres) ${ }^{16}$. Rahel Jaeggi, Amy Allen, Martin Saar et Robin Celikates figurent parmi les représentants de cette tradition.

Pour Martin Saar, la rénovation du projet critique ne peut actuellement être envisagée qu'à partir de la «philosophie sociale» que Honneth distingue de la philosophie politique. La philosophie sociale, comme le propose Saar, a été inventée en tant qu'expression collective pour traiter un large éventail de problèmes résultant de la confrontation entre, d'une part, un ordre social ou politique et, de l'autre, l'ordre subjectif et individuel. De ce point de vue, la philosophie sociale est tournée vers un espace intermédiaire entre société et subjectivité ${ }^{17}$. Cela nous permet d'observer la présence d'une orientation constitutive de la tâche critique de la philosophie sociale, nous permettant de la comprendre comme l'aspect critique de la réflexion philosophique. C'est seulement en ce sens que la Théorie critique de la société peut être comprise comme la réalisation du programme de philosophie sociale ${ }^{18}$. Ricœur avait déjà proposé une orientation constitutive dans son analyse de l'espace sociale en mobilisant les outils de la phénoménologie dans «Hegel et Husserl sur l'intersubjectivité » (1977). Pour conjurer le risque de réification de l'esprit objectif hégélien par rapport au social, Ricœur mobilise la phénoménologie d'autrui explicitée par Husserl dans la «Cinquième méditation» (en la couplant avec la sociologie wébérienne). Ricœur salue en Husserl « (...) son refus sans compromission d'hypostasier les entités collectives et dans sa volonté tenace de les réduire toujours à un réseau d'interactions $\gg{ }^{19}$.

15. F. Fischbach, Manifeste pour une philosophie sociale, Paris, La Découverte, 2009, p. 24.

16. Cf. J.-Ph. Deranty, «Critical Theory », dans M. Bevir (éd.), Encyclopedia of Political Theory, London, SAGE, 2010 ; et C. Corradeti, «The Frankfurt School and Critical Theory », dans Encyclopedia of philosophy, disponible en ligne : http://www.iep.utm.edu/frankfur/.

17. Cf. M. Saar, « Power and Critique », Journal of Power, Vol. 3, N 1 (2010), p. 7-20, p. 7.

18. Cf. Ibid.

19. P. Ricœur, «Hegel et Husserl sur l'intersubjectivité », dans Du texte à l'action. Essais d’herméneutique II, Paris, Seuil, 1986, p. 281-302, p. 301. 
Ainsi, dans ce même article, Ricœur reconnaît « que Husserl lui-même donne deux appuis pour sortir du cercle enchanté de l'idéalisme subjectif $»^{20}$. Le premier est la conviction que le fil conducteur transcendantal est l'objet: "C'est seulement à partir de l'objet déjà constitué que l'on peut, rétroactivement, rétrospectivement, déployer les couches de sens, les niveaux de synthèse, faire apparaître des synthèses passives derrière les synthèses actives $»^{21}$. Le second appui consiste à diriger la même exigence vers la constitution de l'intersubjectivité en tant que constituant du social : « la règle selon laquelle aucune analyse constitutive n'est possible sans le fil conducteur de l'objet ne vaut pas seulement pour la Dingkonstituion mais pour la constitution d'autrui $»^{22}$.

S'il est clair pour nous que la Théorie critique suppose toujours une critique constitutive dans la mesure où il s'agit là de la radicalisation d'une interprétation superficielle à partir de laquelle elle atteint la racine de la problématique sociale, il faut que ce travail soit réalisé dans un horizon perfectible. Telle semble être la conviction de Ricœur, qui essaie de faire dialoguer des perspectives différentes par rapport au social. De la même manière, la quatrième génération de la Théorie critique cherche à faire émerger un dialogue avec d'autres traditions pour que, en passant en revue leurs propres fondements remis en question par les critiques susceptibles de leur être adressées du dehors, elle puisse accomplir la tâche consistant à les radicaliser. La position d'Amy Allen dans The End of Progress en est un bon exemple. Elle critique en effet les fondements de la Théorie critique en s'inspirant de la critique post et dé-coloniale ${ }^{23}$. Elle suppose qu'en remettant en question les propositions de ces représentants à la lumière des défis de la critique post et dé-coloniale, elle contribue à assurer la bonne exécution du travail d'émancipation de la Théorie critique par le biais d'une révision critique de ses propres fondements.

La recherche de l'émancipation et la critique d'orientation constitutive $\mathrm{du}$ social dans un horizon perfectible semblent exprimer les objectifs de la Théorie critique actuelle. D'après Ricœur, ce programme ne peut être réalisé qu'en tant que critique des idéologies. Comme nous le savons, l'une des méthodes les plus importantes de la Théorie critique pour achever l'émancipation est la critique des idéologies de facture marxienne, méthode qui a été reprise par Ricœur dans sa pensée des années 1970. Certains éléments phénoménologiques du traitement par Ricœur de cette méthode peuvent constituer des apports à la Théorie critique.

20. Ibid., p. 288.

21. Ibid., p. 289.

22. Ibid., p. 290.

23. Cf. A. Allen, The End of Progress. Decolonizing the Normative Foundations of Critical Theory, New York, Columbia University Press, 2016. Dans le cadre d'un autre travail, elle utilise également la théorie féministe et celle du genre en tant que dispositifs de critique des fondements de la Théorie critique : A. Allen, «Emancipation without Utopia: Subjection, Modernity, and the Normative Claims of Feminist Critical Theory », Hypatia, Vol. 30, $\mathrm{N}^{\circ} 3$ (2015), p. 513-529. 


\section{La critique généalogique des idéologies}

La critique des idéologies de l'École de Francfort comprend trois caractéristiques, selon Geuss ${ }^{24}$, qui essaie de résumer les aléas de cette méthode entre la première et la deuxième génération de la Théorie critique. En premier lieu, elle conçoit que la critique radicale de la société se révèle inséparable de la critique de l'idéologie dominante. Ainsi, le but de toute recherche portant sur le social doit consister à élaborer une critique de la théorie de la société dont la critique de l'idéologie constituerait une partie intégrale. En deuxième lieu, la critique de l'idéologie ne consiste pas simplement en une critique moralisante : elle est alors une tâche cognitive, une sorte de connaissance. En troisième lieu, la critique de l'idéologie implique des changements fondamentaux du point de vue épistémologique de l'empirisme traditionnel du fait que cette critique possède une structure cognitive qui diverge complètement de celle des sciences de la nature ${ }^{25}$.

La première fois que Ricœur évoque cette méthode critique, c'est dans l'article «Herméneutique et critique des idéologies »" où, tout en se situant dans la troisième génération de la Théorie critique, il développe une réponse au débat entre Gadamer et Habermas concernant l'opposition entre l'herméneutique et la critique des idéologies qui, selon Ricœur, "dépasse considérablement les limites d'une discussion sur le fondement des sciences sociales $»^{27}$. Ce que ce débat met en jeu avant tout, c'est ce qu'il appelle «le geste philosophique de base ${ }^{28}$, qui sert de point de départ à la proposition ricœurienne d'une herméneutique critique comprise comme une herméneutique qui reconnaît en elle-même les conditions d'une critique des idéologies. Dans ce texte, nous trouvons déjà des éléments développés dans ses réflexions postérieures sur l'imaginaire social, car il voit ici, dans l'idée de la variation imaginative de l'ego, « la possibilité la plus fondamentale pour une critique des illusions du sujet $»^{29}$. Lors de ses conférences portant sur la relation entre la science et l'idéologie ${ }^{30}$, entre l'idéologie et l'utopie ${ }^{31}$

24. R. Geuss, The Idea of a Critical Theory. Habermas and the Frankfurt School, Cambridge, Cambridge University Press, 1981.

25. Cf. Ibid., p. 26.

26. P. Ricœur, "Herméneutique et critique des idéologies », dans Du texte à l'action. Essais d'herméneutique II, op. cit., p. 333-378. Publié initialement dans E. Castelli (éd.), Démythisation et Idéologie, Paris, Aubier-Montaigne, 1973, p. 25-64.

27. P. Ricœur, « Herméneutique et critique des idéologies », op. cit., p. 333.

28. Ibid.

29. P. Ricœur, ibid., p. 360.

30. Conférence donnée à l'Université Catholique de Louvain (P. Ricœur, « Herméneutique et critique des idéologies », dans Du texte à l'action. Essais d'herméneutique II, op. cit.).

31. Conférences données à l'Université de Chicago (P. Ricœur, Idéologie et utopie, Paris, Seuil, 1997). 
et sur l'imagination ${ }^{32}$, Ricœur développera l'un de ses apports à la philosophie sociale relatif aux imaginaires sociaux qui, à notre avis, doit être compris en contrepoint de sa méthode phénoménologique. Nous suivons en cela le point de vue de Johann Michel selon lequel l'apport de Ricœur consiste à transférer la fonction des variations imaginatives de la phénoménologie husserlienne à la philosophie de l'action sociale et politique ${ }^{33}$.

Mais avant d'examiner les apports phénoménologiques de Ricœur à cette méthode, notamment, pour la dernière génération de la Théorie critique, nous devons dépasser les critiques formulées par Robin Celikates à l'égard de la philosophie de Ricœur. Celikates est l'un des penseurs de la critique des idéologies et un représentant de la dernière génération de l'École de Francfort. Selon lui, la critique paradigmatique à l'encontre de la tradition de la Théorie Critique, telle qu'elle était pensée par Marx, revêt trois $\operatorname{aspects}^{34}$ : a) la critique de Marx constitue tout à la fois une critique des formes de connaissance et des formes de pratique qui leur correspondent ; b) c'est une critique pratique et émancipatoire parce qu'elle ne cherche pas seulement à comprendre mais aussi à provoquer une transformation du social ; c) Marx suit Hegel en rejetant la dichotomie entre critique interne et externe : ils optent pour la critique immanente. Selon Celikates, la version marxiste de la critique immanente se concentre sur les contradictions internes et les crises d'un ordre social spécifique ainsi que sur son imaginaire social. Mais, pour réactualiser cette critique par rapport aux demandes de notre époque, Celikates propose, en faisant un détour par le tournant pragmatiste de Boltanski, de prendre en considération les capacités critiques des agents : «Seule une théorie sociale qui prend comme point de départ les capacités critiques et les pratiques des agents eux-mêmes peut présenter un cadre viable pour penser à la fois idéologie et critique après le tournant pragmatique $»^{35}$. Or c'est précisément l'orientation constitutive de la critique ricœurienne qui consiste à prendre pour point de départ de l'expérience des agents l'un des grands apports de la phénoménologie à la Théorie critique à travers Ricœur. Boltanski avoue précisément, comme nous l'avons affirmé au début de cet article, s'en être inspiré. Cependant, selon Celikates,

32. Les conférences sur l'imagination demeurent encore inédites (cf. G. Taylor, « Ricœur's Philosophy of Imagination », dans Journal of French Philosophy, Vol. 16, №1-2, 2006, p. 93-104).

33. J. Michel, Ricœur et ses contemporains, Paris, PUF, 2013.

34. R. Celikates, « Karl Marx. Critique as Emancipatory Practice», dans Karin de Boer/Ruth Sonderegger (éd.): Conceptions of Critique in Modern and Contemporary Philosophy, London, Palgrave Macmillan, 2011, p. 101-118, p. 101.

35. «only a social theory that takes the critical capacities and practices of the agents themselves as a starting point can present a viable framework for thinking both ideology and critique after the pragmatic turn" (R. Celikates, «From Critical Social Theory to a Social Theory of Critique: On the Critique of Ideology after the Pragmatic Turn », dans Constellations, Vol. 13, № 1 (2006), p. 21-40, p. 22). 
la pensée de Ricœur fondée sur la médiation symbolique de la réalité présente les distorsions idéologiques comme dérivées ou secondaires. Du point de vue scientifique, cela signifie, aux yeux de Celikates, que la nature interprétative de l'objet de recherche dans le champ des sciences humaines et sociales exclut toute coupure épistémologique qui rendrait possible le passage de l'idéologie à la science et à la critique, conçues comme une réalité séparée ${ }^{36}$. Comme le déclare Celikates :

Mais avec cette rupture, la métacritique herméneutique et ethnologique rejette aussi l'idée même de critique (ou, comme dans le cas de Ricœur, la reformule comme une critique interne qui de manière imaginative s'appuie sur l'explicitation des possibilités cachées du présent). Elle semble ainsi souscrire à la même prémisse fondamentale que Bourdieu : une critique de l'idéologie ne serait possible que d'un point de vue extérieur à la pratique ${ }^{37}$.

Contrairement à ce que pense Celikates, à notre avis, il ne faut pas comprendre l'approche de Ricœur comme une critique interne qui dévoile les possibilités du présent, ni comme appartenant aux groupes des critiques dites herméneutiques ou ethnologiques à partir desquelles une critique des idéologies serait possible seulement du point de vue externe à la pratique de la critique. Bien au contraire, nous défendons la théorie selon laquelle, du point de vue de son soubassement phénoménologique, la critique des idéologies fondée sur le sens ricœurien de l'imaginaire social agit comme une critique immanente et émancipatrice, qui opère comme une critique tout à la fois des formes de connaissance et de leurs formes de critique. Cependant, il faut dépasser le cadre de l'herméneutique pour pouvoir cerner les apports phénoménologiques de Ricœur à la Théorie critique.

Bien que l'on puisse trouver dans l'herméneutique ricœurienne une ressource importante pour la philosophie sociale, nous estimons que sa phénoménologie se révèle plus intéressante au regard de l'orientation constitutive du social. Toutefois, la relation entre la pensée de Ricœur et la philosophie sociale divise les spécialistes. Quelques commentateurs, comme Johann Michel ${ }^{38}$, Anna Borisenkova ${ }^{39}$ et Alain Loute ${ }^{40}$, situent Ricœur dans

\section{Cf. ibid., p. 29.}

37. « With this break, however, the hermeneutic and ethnological metacritique also dismisses the very idea of critique (or, as in the case of Ricour, reformulates it as an internal critique that imaginatively draws on the utopian disclosure of the hidden possibilities of the present). It thereby seems to subscribe to the same fundamental premise as Bourdieu : a critique of ideology would only be possible from a standpoint outside of practice » (ibid., p. 29).

38. J. Michel, « Narrativité, narration, narratologie : du concept ricœurien d'identité narrative aux sciences sociales », Revue européenne des sciences sociales, Vol. 41, N 125 (2003), p. $125-142$.

39. A. Borisenkova, « Narrative Refiguration of Social Events. Paul Ricœur's Contribution to Rethinking the Social », dans Études Ricœuriennes/Ricœur Studies, Vol. I, N ${ }^{\circ} 1$ (2010), p. 87-98. 
l'horizon de la philosophie du langage. Selon cette perspective, son principal apport résiderait à l'intérieur d'une herméneutique de l'identité narrative qui limiterait l'espace social ricœurien, en laissant de côté l'expérience non linguistique. D'autres auteurs, tels que Gonçalo Marcelo ${ }^{41}$ et Suzi Adams ${ }^{42}$, soulignent l'importance pré-prédicative du symbolisme ricœurien en insistant sur l'articulation entre phénoménologie et herméneutique dans la pensée de Ricœur. Or à notre sens, c'est précisément de cette manière que Jaeggi comprend la critique des idéologies de Ricœur.

Jaeggi affirme que l'une des raisons du renouvellement de la critique des idéologies est son potentiel pour identifier les problèmes les plus aigus du débat actuel portant sur la critique sociale ${ }^{43}$. Selon Jaeggi, la critique des idéologies est une critique de la domination livrée « en profondeur» :

Elle agit comme une attaque contre des mécanismes que l'on peut caractériser comme «produisant le cela va de soi» (Ver-selbstverständlichung), donc des mécanismes qui produisent l'impression que les rapports sociaux et les rapports à soi-même existants sont indépassables. De ces mécanismes relèvent les phénomènes de naturalisation - lorsque quelque chose de « fabriqué » socialement est présenté comme un " donné » indépassable -, mais aussi des phénomènes comme ceux de l'universalisation du particulier, qui a une fonction si importante dans l'analyse marxienne du mécanisme de légitimation de la société bourgeoise. La critique de l'idéologie est donc une critique de la domination en tant que critique des mécanismes produisant ce qui va de soi et, inversement, en tant que déchiffrement de ces mécanismes comme des mécanismes de domination ${ }^{44}$.

La critique des idéologies part ainsi des contradictions internes d'une situation donnée, révélées dans la compréhension du monde des individus singuliers. En ce sens, selon Jaeggi, elle « repose toujours sur une forme d' « herméneutique du soupçon», comme la nomme Paul Ricœur » ${ }^{45}$, et sur l'auto-interprétation des formations sociales des individus et de leurs intérêts.

40. A. Loute, "Philosophie Sociale et Reconnaissance mutuelle chez Paul Ricœur», in Affectivité, imaginaire, création sociale, Raphaël Gély et Laurent Van Eynde (éd.), Bruxelles, Facultés Universitaires Saint-Louis, 2010, p. 125-147.

41. G. Marcelo, «Making Sense of the Social. Hermeneutics and Social Philosophy », dans Études Ricœuriennes/Riccur Studies, Vol. 3, № 1 (2012), p. 67-85.

42. S. Adams, «On Ricœur's Shift from a Hermeneutics of Culture to a Cultural Hermeneutics », dans Études Ricœuriennes/Ricœur Studies, Vol. 6, № 2 (2015), p. 130-153.

43. R. Jaeggi, «Rethinking Ideology», dans B. De Bruin et C. Zurn (éds.), New Waves in Political Philosophy, London, Palgrave Macmillan, 2009, p. 63-86, p. 63 (pour une version française plus brève et préliminaire de ce texte: R. Jaeggi, "Qu'est-ce que la critique de l'idéologie? », Actuel Marx, Vol. 1, № 43 (2008), p. 96-108). Nous utilisons la version en anglais qui est plus complète.

44. R. Jaeggi, « Qu'est-ce que la critique de l'idéologie ? », op. cit., p. 97.

45. R. Jaeggi, « Rethinking Ideology », op. cit., p. 69. 
Jaeggi a pour ambition d'accomplir une « revitalisation de la critique de l'idéologie comme forme de critique sociale ${ }^{46}$, la philosophe défend l'aspect normatif de la critique tout en interprétant la critique des idéologies comme une critique immanente. Selon Jaeggi, «l'une des raisons pour lesquelles le projet de renouvellement de la critique de l'idéologie pourrait en effet "valoir la peine" (...) est qu'il nous permettrait de voir certains problèmes très aigus du débat actuel sur les formes de critique sociale sous un angle nouveau ${ }^{47}$. En ce sens, la critique des idéologies est un type spécifique de critique immanente, à savoir celle qui croise une perspective externe avec une perspective interne :

Je propose que, en tant que type spécifique de critique immanente, la critique de l'idéologie aille au-delà de l'alternative souvent discutée mais peu productive de la critique externe et interne. À savoir, qu'elle s'oppose aux tentatives de déterminer les normes de la critique « extérieure » (et évite ainsi la critique hégélienne de la moralité du « simple devoir »), mais que d'autre part, elle n'est pas entièrement dépendante des ressources éthiques et morales d'une communauté donnée : elle possède un certain moment transgressif à propos de ces ressources. À ce trait est lié un deuxième aspect, à savoir que la critique de l'idéologie se situe de manière intéressante entre les positions «antinormatives» de la philosophie politique et les positions que l'on pourrait appeler «normatives». Face aux signes de tension et d'épuisement qui caractérisent actuellement ce conflit, la critique de l'idéologie pourrait être reconstruite comme une position qui revendique un autre type de normativité, au-delà de ces deux alternatives ${ }^{48}$.

D'après Jaeggi, la base normative de la critique des idéologies n'est pas externe mais immanente. À son tour, la critique des idéologies, selon Jaeggi, peut répondre à la tension entre le plan subjectif et celui objectif de l'espace social, ainsi qu'entre ses plans ontique et ontologique. De la même manière, Ricœur renvoie le sens des idéologies à la dimension constitutive de

46. « Revitalization of the critique of ideology as a form of social critique » (ibid., p. 63).

47. « one reason why the project of renewing the critique of ideology might indeed be "worth the trouble" (...) is that it would allow us to see some very acute problems of the current debate about forms of social critique in a new light» (ibid., p. 63).

48. "I propose that, as a specific type of immanent critique, the critique of ideology actually goes beyond the often discussed but not very productive alternative of external and internal critique. Namely, it opposes attempts to determine the standards of critique "externally" (and thus avoids the Hegelian critique of the morality of the "mere ought"), yet on the other hand, it is not wholly dependent on the ethical and moral resources of a given community either: it possesses a certain transgressive moment regarding those resources. Linked to this trait is a second aspect: the critique of ideology is interestingly positioned between the "antinormative" positions in political philosophy, and those positions one might correspondingly call "normative". In the face of the signs of strain and exhaustion that this conflict currently exhibits, the critique of ideology might be reconstructed as a position that lays claim to a different kind of normativity, beyond those two alternatives » (ibid., p. 64). 
l'expérience pré-linguistique de la subjectivité au sein de laquelle l'imagination occupe une place centrale: la structure symbolique fondamentale de l'action est la vie sociale qui n'est pas nécessairement négative. La critique des idéologies pourrait se réaliser à l'égard d'une utopie face à laquelle l'idéologie prend sa négativité. La phénoménologie donne à Ricœur la possibilité de légitimer le geste philosophique en direction d'une expérience irréductible à ses moyens d'explicitation. À notre avis, lorsqu'un tel geste est effectué, nous pouvons parler d'une émancipation qui se réalise dans une dimension de l'action plus fondamentale que la raison, à savoir dans l'imagination.

Lorsqu'il se préoccupe de la critique des idéologies, il croise la tradition marxienne de l'émancipation avec les apports phénoménologiques sur l'imagination pour établir les bases de sa critique herméneutique. La méthode phénoménologique de description des expériences des individus occupe ici une place fondamentale dans la dialectique entre distanciation et appartenance rendant possible la critique des idéologies. C'est ainsi qu'avec la méthode de la critique des idéologies, le projet émancipatoire se déploie comme une mise à distance critique des idéologies par les utopies. Afin de bien saisir ce point, il faut porter notre attention sur les écrits encore inédits sur l'imagination que Ricœur avait rédigés pour un groupe de conférences données à Chicago en 1975 sous le titre « Lectures on Imagination $» 4$.

La distinction entre l'imagination reproductive - qui a été privilégiée d'Aristote à Kant et de Kant à Sartre - et l'imagination productive - dont Ricœur veut proposer la théorie - domine la totalité de ces textes inédits ${ }^{50}$. D'après Ricœur, il existerait ainsi quatre domaines de l'imagination productive $^{51}$. En premier lieu, dans le domaine de l'imagination sociale et culturelle, l'utopie est la fiction de l'imagination productive et l'idéologie, l'imagination sociale reproductive ${ }^{52}$. La dialectique de l'idéologie et de l'utopie est exclusivement traitée dans un autre groupe de conférences données à Chicago à cette même époque et dont le manuscrit a été publié pour la première fois en anglais ${ }^{53}$. En second lieu, nous trouvons le domaine

49. «Ces conférences sont le seul endroit où l'on trouve un cadre structurel plus complet de l'argumentation de Ricœur, qui passe de l'analyse de l'imagination reproductive à celle de l'imagination productive» ("These Lectures provide the only place where we find a more complete structural framework of Ricœur's argument as it moves from an analysis of reproductive to productive imagination », G. Taylor, "Ricœur's Philosophy of Imagination », op. cit., p. 93).

50. Ibid.

51. Ibid., p. 94.

52. Ibid.

53. Taylor avoue que « la grande fascination qu'exerçaient les deux séries de conférences était donc en partie due au fait qu'elles avaient en commun le thème de l'imagination » (« Part of the great fascination, then, of hearing both sets of lectures was the commonality between them of the theme of imagination », ibid., p. 94). 
épistémologique de l'imagination productive et, en troisième lieu, le domaine poétique de l'imagination productive ${ }^{54}$. Enfin, le concept d'imagination productive est traité à partir du domaine du symbole religieux $^{55}$. Ces réflexions sont à la base de l'approche ricœurienne du monde social, à travers les concepts d'idéologie et d'utopie.

Dans L'Idéologie et l'utopie, Ricœur souligne la fonction d'intégration qui projette l'idéologie dans l'espace social, fonction qui la rend corrélative à l'utopie, face à laquelle l'idéologie acquiert son aspect négatif. Une idéologie négative ou déformante est celle qui se fonde sur les représentations (Vorstellungen) de l'espace social. C'est Marx qui a dénoncé les problèmes de cette idéologie, en ayant recours à la méthode de la critique des idéologies $^{56}$. Mais la science marxiste est elle-même une idéologie qui a l'intention de présenter la réalité telle qu'elle est (Darstellung). L'utopie de Marx se figure dans le futur et produit, à partir de ce diagnostic, l'image de l'émancipation. C'est ici que nous trouvons un premier aspect phénoménologique: la compréhension de l'utopie comme une fonction de l'imagination productrice. Ainsi, Ricœur affirme : «Si je devais rapporter cette structure de l'utopie à la philosophie de l'imagination, je dirais qu'elle se rapproche des variations imaginaires autour d'une essence que propose Husserl $»^{57}$. L'utopie effectue en effet une neutralisation imaginative et des variations imaginaires autour de questions telles que celles de la société, du pouvoir, du gouvernement, de la famille, de la religion ${ }^{58}$. C'est de cette manière que Ricœur finit par définir/caractériser l'utopie à partir de sa fonction consistant à introduire un «nulle part» dans la constitution du social $^{59}$. Il existe cependant un élément phénoménologique plus fondamental encore, qui concerne la motivation de la méthode utilisée :

Mon analyse de l'utopie et de l'idéologie est une analyse régressive de la signification. Elle n'est pas une analyse des idéal-types, mais plutôt une phénoménologie génétique au sens proposé par Husserl dans les Méditations cartésiennes. Cette méthode nous permet d'atteindre le niveau de la description sans nous situer hors des connexions qui relient l'idéologie et l'utopie. Une phénoménologie génétique s'efforce de creuser sous la surface de la signification apparente jusqu'aux significations plus fondamentales. L'effort consiste à reconnaître la revendication d'un concept, qui est à première vue un simple outil polémique, pour tenter de légitimer le concept ${ }^{60}$.

\section{Cf. ibid.}

55. Ibid.

56. P. Ricœur, L'Idéologie et l'utopie, op. cit., p. $103 \mathrm{~s}$.

57. Ibid., p. 36.

58. Ibid., p. 37.

59. Ibid.

60. Ibid., p. 408. 
Selon Ricœur, aussi bien l'idéologie que l'utopie - qui sont les deux expressions de l'imagination culturelle et sociale - possèdent un côté positif et un autre négatif; un rôle constructif et un autre destructeur; une dimension constitutive et une autre pathologique. Le fait que ce soit les aspects négatifs qui apparaissent tout d'abord n'est, selon Ricœur, qu'une indication pour procéder de manière régressive, « en partant de la surface des choses ». Une telle exigence généalogique et immanente est défendue par la dernière génération de la Théorie critique.

Dans la perspective généalogique, la critique des idéologies rejoint l'orientation constitutive de la Théorie critique. Cet aspect s'avère évident d'après une lecture de Saar qui met également en avant les puissances de l'imaginaire social. Il comprend l'orientation constitutive comme une critique du pouvoir, qui peut être saisie en tant que domination ou du point de vue ontologique. Selon Saar, cette double dimension de la critique a été donnée dans la généalogie nietzschéenne. Ainsi, dans un texte qui distingue les différentes notions de pouvoir présentées dans La Généalogie de la morale, Saar identifie un premier sens dans l'examen nietzschéen des valeurs culturelles et morales (ce qui peut être compris comme des combats réels de pouvoir historiquement sédimentés en valeurs de pouvoir symbolique). Nietzsche s'attarde précisément sur certaines luttes des dominés et des êtres en situation d'esclavage. Saar affirme néanmoins que d'autres dimensions du pouvoir apparaissent lorsque nous considérons sa conception de la "volonté de puissance ${ }^{61}$, qui constitue une dimension subliminale (ou imaginaire) du pouvoir, qui produit des identités. En se penchant sur cette dimension de la généalogie, Saar soutient que, dans la rhétorique de Nietzsche, se développe une critique du pouvoir en tant que virtualisation de l'histoire ${ }^{62}$. La pertinence de la compréhension du travail de la Théorie critique en tant que « critique du pouvoir » est saisie dans l'espace de tension entre la dimension subjective et celle objective de l'espace social, ainsi que dans la possibilité de rendre compte de manière critique des plans ontiques et ontologiques de cet espace, ce qui permet de formuler les exigences généalogiques de la Théorie critique.

Ricœur a développé cette orientation généalogique grâce à sa lecture des Méditations cartésiennes de Husserl, texte auquel il consacrera deux articles dans les années 1960 inclus dans À l'école de la phénoménologie. Nous sommes convaincue que sa lecture de Husserl a eu fortement influencé sa conception de l'espace social. La compréhension de l'idéologie en tant que critique d'orientation constitutive dans la pensée de Ricœur doit se faire à partir du proche rapport existant entre Lectures on Imagination et Lectures on Ideology and Utopia. N'oublions pas que ces deux groupes de conférences ont été donnés durant la même semaine à l'université de Chicago. Il affirme justement: «ma conviction, ou tout au moins mon

61. Cf. M. Saar, «Understanding Genealogy : History, Powers, and the Self », dans Journal of the Philosophy of History, vol. 2 (2008), p. 295-314, p. 303.

62. Cf. ibid., p. 312. 
espoir, est que la dialectique entre idéologie et utopie pourra apporter quelque lumière à la question non résolue de l'imagination comme problème philosophique ${ }^{63}$. Précisément, selon Ricœur, en tant que distorsion ou dissimulation, la conception de l'idéologie de la Théorie critique ne s'avère pas assez radicale. Il faudrait l'aborder depuis une analyse de l'imaginaire qui dévoile la place fondamentale du sujet, dernier point qui nous semble très proche de la préoccupation de Celikates pour les agents dans la perspective critique du social.

La phénoménologie permet à Ricœur de proposer une notion de critique des idéologies de cette ampleur. La critique radicale sera fondée, selon Ricœur, sur la base d'une large conception de l'imagination: "C'est pourquoi je cherche une fonction de l'idéologie plus radicale que celle de distorsion ou de dissimulation. La fonction de distorsion couvre seulement une petite surface de l'imagination sociale, tout comme les hallucinations et les illusions constituent seulement une part de notre activité imaginative en général $»^{64}$. Comment effectuer cette extension imaginative en partant de la notion d'idéologie? Ricœur nous propose de nous pencher sur le paradoxe de Mannheim :

Un des moyens de préparer cette extension radicale est de se pencher sur ce que certains auteurs américains ont appelé le paradoxe de Mannheim, qui résulte de son observation du développement du concept marxiste d'idéologie. Le paradoxe réside dans l'impossibilité d'appliquer le concept d'idéologie à lui-même. En d'autres termes, si tout ce que nous disons est biaisé, si tout ce que nous disons représente des intérêts que nous ne connaissons pas, comment avoir une théorie de l'idéologie qui ne soit pas elle-même idéologique? La réflexion du concept d'idéologie sur lui-même est la source de ce paradoxe ${ }^{65}$.

Ricœur s'adresse à Marx. Avant de se concentrer sur l'Idéologie allemande, livre fondateur de la critique des idéologies, il commente L'Introduction à la Critique de la philosophie du droit de Hegel et les Manuscrits de 1844. À partir de ces deux ouvrages, il défend une lecture humaniste du jeune Marx. De la même manière que dans sa lecture de Husserl des Ideen I, dans laquelle Ricœur met l'accent sur l'imagination, dans sa lecture de Marx, l'importance de l'imagination s'avère explicite. Par exemple, dans l'exposition du concept d'aliénation, il fait référence à la mention de l'imagination faite par Marx pour caractériser comment, de la même manière que l'activité de l'imagination humaine agit sur l'individu indépendamment de lui-même dans la religion, l'activité de l'ouvrier ne constitue plus non plus son activité propre: " J'insiste à nouveau sur le terme "imagination". Ici, ce n'est pas Einbildung mais Phantasie: autrement dit, l'imagination comme fantaisie plus que comme fiction ${ }^{66}$. Selon la

63. P. Ricœur, L'idéologie et l'utopie, op. cit., p. 17.

64. Ibid., p. 18.

65. Ibid.

66. Ibid., p. 69. 
lecture de Ricœur, Marx se réfère à l'imagination au sens large, à celle qui, en tant qu'activité propre à l'homme, peut devenir un foyer d'aliénation, caractérisation toutefois seulement possible dans l'horizon de l'humanisme de Marx :

L'humanisme du jeune Marx, rejeté par les structuralistes (ainsi que nous le verrons dans les chapitres suivants), apparaît très clairement dans ce passage. Une telle description n'a aucun sens si ce n'est pas l'individu - dans son activité propre - qui se trouve affecté, blessé et détruit par l'aliénation. À ce stade au moins de ses écrits, Marx revendique un rôle fondamental pour l'activité propre de l'individu ${ }^{67}$.

Ricœur fait reposer son interprétation de l'Idéologie allemande sur cette conception de l'individu. À la différence des lectures structuralistes, qui distinguent idéologie et science, afin de résoudre le paradoxe de Mannheim, Ricœur va opposer idéologie à réalité mais dans le même sens que l'imaginaire s'oppose à la réalité, comme celle qui, en se différenciant de la réalité, la constitue : "Dans l'Idéologie allemande, l'idéologique est l'imaginaire en tant qu'il s'oppose au réel. Par conséquent, la définition du concept d'idéologie dépend de ce qu'est la réalité - classe ou individu - avec laquelle elle contraste ${ }^{68}$. Deux lectures opposées émergent alors : d'un côté, pour les structuralistes, la réalité sera la classe ; d'un autre côté, pour les humanistes, il s'agira de l'individu. À ce niveau-là, Ricœur dira que le texte nous autorise à maintenir l'ambigüité et il nous propose l'image du canard/lapin de Wittgenstein en précisant que : «L'œuvre ressemble à l'image du canard/lapin chez Wittgenstein (sauf que dans ce cas je ne sais pas ce que pourrait être le lapin !): on peut la lire comme un texte sur les individus réels dans leur vie réelle ou bien comme un texte sur les classes, dans le vocabulaire de la production et non plus dans celui de la vie ${ }^{69}$.

L'approche finit toutefois par accorder une préférence à la lecture humaniste. Tout d'abord, Ricœur comprend le matériel et le réel comme des synonymes, de la même manière que l'idéal et l'imaginaire ${ }^{70}$. Ricœur fait ici appel au passage suivant de l'Idéologie allemande :

Les prémisses dont nous partons ne sont pas des bases arbitraires, des dogmes ; ce sont des bases réelles dont on ne peut faire abstraction qu'en imagination. Ce sont les individus réels, leur action et leurs conditions d'existence matérielles, celles qu'ils ont trouvées toutes prêtes, comme aussi celles qui sont nées de leur propre action. Ces bases sont donc vérifiables par voie purement empirique ${ }^{71}$.

67. Ibid.

68. Ibid., p. 105.

69. Ibid.

70. Ibid., p. 108.

71. Marx, L'Idéologie allemande, cité en : ibid. 
Ricœur fait remarquer que lorsque les prémisses sont réelles, ce qui s'oppose alors à elles, ce sont l'abstraction et l'imagination ${ }^{72}$. Ensuite, les distorsions apparaissent là où nous oublions que les produits de l'abstraction et de l'imagination sont précisément une production. L'exemple de Marx visant à illustrer cette inversion est célèbre dans l'exemple de la camera obscura: «Et si, dans toute l'idéologie, les hommes et leurs rapports nous apparaissent placés la tête en bas comme dans une camera obscura, ce phénomène découle de leur processus de vie historique, absolument comme le renversement des objets sur la rétine découle de son processus de vie directement physique $\gg{ }^{73}$. Selon Ricœur, la camera obscura n'est rien d'autre qu'une métaphore. Ricœur critique en ce sens la lecture d'Althusser qui, en s'inspirant de la philosophie de la science de Bachelard, préfère parler de coupure épistémologique que d'inversement de la camera obscura: «une image inversée reste toujours la même » ${ }^{74}$. De la même manière que Ricœur va critiquer Mannheim dans la partie consacrée à l'utopie dans ses Lectures, il pense qu'Althusser ne sort pas de la demande d'efficacité et du déterminisme du cadre conceptuel du marxisme ${ }^{75}$. D'un autre côté, selon Ricœur, Althusser rapporte le vécu à l'imaginaire : «Une idéologie est à la fois vécu et imaginaire, elle est le vécu comme imaginaire ${ }^{76}$. Si l'on considère que, selon Althusser, il n'existe pas d'individus réels dans la base matérielle, on peut identifier avec Ricœur ${ }^{77}$ une incongruence dans le fait qu'Althusser parle du vécu comme de rapports réels et d'individus réels.

Ricœur nous présente une lecture plus cohérente de la théorie marxienne de l'idéologie. Il relie idéologie et critique et déclare que le concept d'idéologie (Vorstellung) est analogue à celui d'imaginaire et s'oppose au réel. Par conséquent, sa définition dépend de ce que nous appelons le réel (classe ou individu). Si, d'après Marx, l'idéologique s'oppose au réel et non à la science, selon Ricœur, les marxistes postérieurs à Marx l'ont plutôt compris en opposition à la science. Ici, nous pouvons percevoir un paradoxe : la science marxiste ne serait-elle pas elle-même inscrite sur le plan idéologique? S'il s'agit d'une région d'idées, pourrait-elle en venir à se faire face à elle-même ?

Ricœur interprète qu'en tant que Vorstellungen, les idées sont ces représentations dans lesquelles nous nous concevons et non la manière à partir de laquelle nous travaillons et dont nous sommes faits. En considérant de telles représentations, qui constituent notre conscience, comme ce qui se trouve au départ et non comme un produit d'individus, une inversion se

72. Ibid.

73. Marx, L'Idéologie allemande, cité en : ibid., p. 115.

74. Ibid., p. 116.

75. Cf. ibid., p. 153.

76. Ibid., p. 188.

77. Cf. ibid. 
produit. Ainsi, ces fantômes de la conscience ont été placés comme ceux contre lesquels nous nous inclinons. Voilà l'inversion idéologique dénoncée par Marx, selon Ricœur, du concept d'aliénation, depuis l'Introduction à la critique de la philosophie du droit de Hegel, bien que cette expression ne soit pas utilisée, ainsi que dans les Manuscrits de 1844. De la même manière, Ricœur énonce que l'idéologie critiquée par Marx croit pouvoir modifier la vie des hommes en changeant la pensée, en mettant en évidence la figure de l'investissement, puisque cette idéologie évoluera toujours en fonction des représentations, car elle dépend de la conscience et de sa modification. Face à cela, Ricœur nous exhorte à faire attention au matériel ou, ce qui revient au même, au réel : les individus réels, leur activité et les conditions matérielles dans lesquelles ils vivent, de même que les conditions produites par leur propre activité. Les hommes produisent donc leurs conditions et en dépendent, même si la nature de l'individu prévaut toujours. Nous pouvons donc éclairer le choix de Ricœur par la lecture humaniste, à savoir que ce sont les individus réels qui constituent le fondement du réel et non la classe comme la lecture structuraliste le propose.

L'idéologique, qui, chez Ricœur, ne s'oppose pas à la science, est justement constitué par des représentations (Vorstellungen) et il s'agit d'un concept si large qu'il n'a pas nécessairement de signification négative. Il faut donc comprendre l'idéologique simplement par son opposition au réel ou au matériel (wirklich). Grâce à cette lecture, Ricœur peut fournir une réponse satisfaisante au paradoxe de Mannheim. D'après Ricœur, qui s'inspire de Geertz, la science proposée par Marx serait elle aussi un type d'idéologie, mais une idéologie au sens propre, qui repose sur un langage de la vie réelle: "qui préexiste à toutes les distorsions, une structure symbolique de l'action absolument première et incontournable $\gg{ }^{78}$.

Cependant, en ce qui concerne le langage de la vie, nous ne parlerions plus de Vorstellung en tant que représentation, mais de Darstellung comme présentation ${ }^{79}$. C'est en cela que réside, selon Ricœur, le caractère descriptif et indéformable de la science proposée par Marx et de l'idéologie utilisée dans l'exposition de L'Idéologie allemande elle-même. Ainsi, la distorsion que Marx dénonce vis-à-vis de l'idéologie renvoie à une forme inadéquate de cette dernière, qui consiste à oublier que nos représentations (Vorstellungen) sont une production et que nous subissons les effets d'une inversion. L'idéologique serait constitué par la moralité, la religion, la métaphysique et toute la sphère culturelle en général. $\mathrm{Si}$, avec cet oubli,

78. Ibid., p. 115.

79. Taminiaux accorde également une grande importance à la distinction entre Darstellung et Vorstellung, dans sa lecture de Marx (cf. J. Taminiaux, «Empirisme et spéculation chez Marx », dans Recoupements, Brussels, Ousía, 1982, p. 49-73) ; cependant, Ricœur, dans un autre texte (cf. P. Ricœur, « Science et idéologie », in Revue Philosophique de Louvain, Vol. 72, $\mathrm{N}^{\circ} 14,1974$, p. 344), affirme que la lecture de Taminiaux diffère de la sienne, car la lecture ricœurienne ne mènera pas Marx à la ligne de l'ontothéologie, contrairement à Taminiaux qui, selon lui, s'aligne trop sur Feuerbach. 
l'investissement intervient, une réparation sera donnée à la recherche d'un ordre dans lequel l'individu occupera l'espace primordial de protagoniste de la moralité, de l'histoire en général, et en tant que producteur de la sphère culturelle. Selon Ricœur, comme nous le verrons dans le chapitre suivant, cela ne peut se produire que par le biais d'une phénoménologie de la fiction qui, en tant que critique, distingue l'aspect constituant de l'imagination du déformant qu'est la fuite du réel :

[T] out se passe comme si l'imagination sociale, ou l'imagination culturelle, opérant à la fois de manière constructrice et de manière destructrice, était à la fois une confirmation et une contestation de la situation présente. Peut-être est-ce une hypothèse féconde que la polarité de l'idéologie et de l'utopie est en rapport avec les différentes figures de la non-congruence caractéristiques de l'imagination sociale. Et peut-être que les côtés respectivement négatifs et positifs de chacune des deux notions sont en rapport mutuel ${ }^{80}$.

Ricœur a recours, afin de montrer l'aspect constitutif de l'idéologie, à la notion d'appartenance qui fonde celle d'intégration : " (...) tout savoir objectivant sur notre position dans la société, dans une classe sociale, dans une tradition culturelle, dans une histoire, est précédé par une relation d'appartenance que nous ne pourrons jamais entièrement réfléchir $»^{81}$. Ricœur dirait que face à tout investissement, de quelque type qu'il soit, nous devons tout d'abord souligner notre appartenance aux groupes sociaux euxmêmes. En ce sens, la critique ricœurienne des idéologies se révèle plus radicale que celle qui critique la domination. Ricœur rejoint ici les demandes de la critique ontologique du pouvoir de Saar sans pour autant laisser de côté la critique des idéologies. Parallèlement, dans les Cinq articles sur le renouveau, Husserl rapporte l'appréhension de l'eidos de la collectivité à la fonction d'intégration, car une unité de vie collective se constitue à travers toute existence individuelle ${ }^{82}$.

Aussi bien Ricœur que Husserl ont atteint le même horizon concernant la relation entre l'imagination et la constitution de l'espace social, même si Ricœur n'a pas vu ladite relation dans la phénoménologie husserlienne : «C'est dans ce travail sur l'imaginaire social que se médiatisent les contradictions qu'une simple phénoménologie de l'imagination individuelle doit laisser à l'état d'apories ${ }^{83}$. C'est pour cette raison que la critique des idéologies, qui relève du social, est au fond l'authentique réalisation de la phénoménologie. De toute façon, il est clair que Ricœur a trouvé, dans le concept husserlien des variations imaginaires, l'inspiration nécessaire pour parcourir son propre cheminement vers l'imaginaire social.

80. P. Ricœur, L'Idéologie et l'utopie, op. cit., p. 17.

81. P. Ricœur, « Science et idéologie », op. cit., p. 352.

82. Cf. E. Husserl, Aufsätze und Vorträge. 1922-1937, Hua XXVII, T. Nenon et H.R. Sepp (eds.), The Hague, Netherlands, Kluwer Academic Publishers, 1988, p. 48.

83. P. Ricœur, « L'imagination dans le discours et dans l'action », Du texte à l'action, op. cit., p. 213-236, p. 236. 
Une influence husserlienne dans la conception ricœurienne de l'idéologie est la compréhension de la finitude de notre pensée elle-même qui rend la réflexion absolue impossible. Cette finitude implique justement que la tâche de la critique des idéologies est infinie. La réflexion de Ricœur sur cet aspect est influencée par le traitement par Eugen Fink des concepts opératoires de la phénoménologie. Ricœur affirme que :

Quelqu'un comme Eugen Fink, par exemple, l'a aussi souligné, dans son article sur les concepts thématiques et les concepts opératoires. Nous ne pouvons pas penser tout ce qui est à l'œuvre dans notre pensée. Nous pensons par des concepts, au moyen de concepts. C'est pourquoi nous ne pouvons parvenir à une totale transparence de la pensée. Nous pouvons penser à quelque chose, thématiquement, mais pour ce faire nous devons mettre en œuvre des concepts qui ne sont pas thématisés, au moins tant que nous nous en servons. Dans mon propre langage, je dirais qu'une réflexivité absolue est impossible. Nous ne pouvons parvenir qu'à une réflexion partielle. Il est donc possible qu'une part importante du concept d'idéologie signifie que nous ne pouvons réfléchir tous nos concepts ${ }^{84}$.

En ce sens, la définition ricœurienne de cette méthode critique répond aux intérêts critiques de la dernière génération de la Théorie critique qui essaie, comme nous l'avons dit, de revisiter ses principes, méthodes et fondements en établissant des dialogues avec d'autres traditions de pensée. De plus, dans le concept d'idéologie, Ricœur développe un véritable apport phénoménologique à la tradition de la Théorie critique, à savoir que l'utopie constituerait le côté productif de l'imagination sociale, là où la critique rencontre son foyer non-déformant.

La phénoménologie de l'imaginaire social de Paul Ricœur est donc un riche foyer conceptuel pour la critique du social d'un point de vue constitutif. Comme nous l'avons établi, l'approche phénoménologique de Ricœur donne un angle constitutif du social à la critique émancipatrice de la Théorie critique, notamment en ce qui concerne la constitution de l'intersubjectivité. À cela s'ajoute une approche phénoménologique de la critique des idéologies, d'orientation génétique et de préoccupation humaniste. Au fond, la phénoménologie de Ricœur nous apprend que ce sont les agents ou les subjectivités individuelles qui constituent le fondement $\mathrm{du}$ social. Toute Théorie critique doit absolument défendre cette première évidence, bien que de manière perfectible et infinie, à une époque marquée par de multiples catastrophes. 